\title{
NOTICIAS
}

\section{Presentación de resultados de la Encuesta Nacional de Nutrición 1999. Estado nutricio de niños y mujeres en México}

El 19 de febrero del año en curso se presentó el libro titulado "Encuesta Nacional de Nutrición 1999. Estado nutricio de niños y mujeres en México, ${ }^{11}$ el cual describe, analiza e interpreta los resultados de la segunda Encuesta Nacional de Nutrición, realizada por el Instituto Nacional de Salud Pública en colaboración con el Instituto Nacional de Estadística, Geografía e Informática, entre 1998 y 1999. La Encuesta fue financiada principalmente por la Secretaría de Salud.

La reunión en la que se hizo la presentación fue convocada por el Dr. Julio Frenk Mora, Secretario de Salud, en el auditorio "Miguel E. Bustamante" de la Secretaría de Salud. La síntesis de los resultados fue expuesta por los doctores Jaime Sepúlveda Amor, Director General del Instituto Nacional de Salud Pública y Juan Rivera Dommarco, Director del Centro de Investigación en Nutrición y Salud, quien coordinó al grupo que realizó la encuesta. Participaron con sus comentarios dos prestigiados investigadores en el campo de la nutrición: los doctores Silvestre Frenk y Reynaldo Martorell.
La Encuesta Nacional de Nutrición estudió una muestra probabilística de alrededor de 21000 hogares, representativa del ámbito nacional, de zonas urbanas y rurales, y de cuatro Regiones: Norte, Centro, Ciudad de México y Sur.* Se estudiaron tres grupos de edad: niños en edad prescolar (menores de cinco años), niños en edad escolar (entre 5 y 11 años), y mujeres entre 12 y 49 años de edad. Se presenta una síntesis de los resultados por grupo de edad.

Niños en edad prescolar (menores de cinco años)

Los principales problemas de nutrición en este grupo de edad fueron la desnutrición crónica o retardo en estatura, la anemia y las deficiencias de vitaminas y minerales. Dichos problemas tienen efectos negativos en el desarrollo mental y

\footnotetext{
* Norte (Baja California, Baja California Sur, Coahuila, Chihuahua, Durango, Nuevo León, Sonora, Tamaulipas); Centro (Aguascalientes, Colima, Guanajuato, Jalisco, México -excluye municipios y localidades conurbadas a Ciudad de México-, Michoacán, Morelos, Nayarit, Querétaro, San Luis Potosí, Sinaloa, Zacatecas); Ciudad de México (incluye el Distrito Federal y los municipios conurbados del Estado de México), y Sur (Campeche, Chiapas, Guerrero, Hidalgo, Oaxaca, Puebla, Quintana Roo, Tabasco, Tlaxcala, Veracruz, Yucatán).
}

en la respuesta inmunológica, lo que conduce a un aumento en el riesgo de enfermar y de morir. Además, tienen efectos adversos a largo plazo como menor desempeño escolar e intelectual y menor rendimiento físico en escolares, adolescentes y adultos.

En México casi uno de cada cinco niños menores de cinco años tiene desnutrición crónica, lo que se considera un importante problema de salud pública. Se encontraron diferencias notables entre los porcentajes de niños con desnutrición crónica en zonas urbanas y rurales y en las distintas Regiones. Así, mientras el porcentaje de niños con desnutrición crónica fue de $32.3 \%$ para las zonas rurales, en las zonas urbanas fue de $11 \%$. En la Región Sur, la más pobre, se encontró el porcentaje más elevado de niños con desnutrición crónica $(29.2 \%)$, seguida de la Región Centro (14.5\%) y la Ciudad de México (13.1\%); el menor porcentaje correspondió a la Región Norte $(7.1 \%)$. Los porcentajes de desnutrición crónica por edad indican que ésta ocurre durante la gestación y en los dos primeros años de vida, etapa considerada como la ventana de oportunidad para evitar que los niños se desnutran, procurándoles una buena alimentación y una adecuada atención de la salud.

La anemia (concentración baja de hemoglobina en sangre), causada 
en parte por deficiencias de hierro y de algunas vitaminas, es otro problema de salud pública en los menores de cinco años. El 27.2\% de los niños de este grupo presentaron anemia. Los porcentajes más elevados se encontraron en niños de entre 12 y 23 meses de edad alcanzando, para el ámbito nacional, $48.8 \%$, lo que indica que para prevenirles la anemia el grupo de menores de dos años debe recibir atención prioritaria.

Las principales deficiencias de vitaminas y minerales se presentaron en los menores de cinco años y fueron la de hierro, en más de la mitad, y las de zinc, vitamina A y vitamina $C$, en alrededor de una tercera parte de estos niños.

\section{Niños en edad escolar (5 a 11 años)}

Los principales problemas de nutrición en este grupo de edad fueron la anemia, varias deficiencias de vitaminas y minerales y la obesidad. La anemia y las deficiencias de vitaminas y minerales afectan la capacidad de aprendizaje del escolar y aumentan su riesgo de enfermar; mientras que la obesidad tiene efectos a largo plazo, aumentando el riesgo de diabetes, enfermedades cardiovasculares y otras enfermedades crónicas en la edad adulta. Cerca de $20 \%$ de los niños en edad escolar tienen anemia; en la Ciudad de México se encontró el dato más bajo, $11 \%$. Alrededor de un tercio de los niños de esta edad presentaron deficiencias de hierro y de vitamina $C$, y cerca de $20 \%$ tuvieron deficiencias de zinc y de vitamina A. Además, la obesidad es un problema frecuente en este grupo de edad. El 18.8\% de los escolares fueron clasificados como con sobrepeso u obesidad. Los datos más altos, cercanos a $25 \%$, se encontraron en la Ciudad de México y la Región Norte. En las zonas urbanas las cifras fueron superiores a $20 \%$, mientras que en las zonas rurales fueron cercanas a $10 \%$.

\section{Mujeres entre 12 y 49 años}

Los principales problemas de nutrición en las mujeres fueron la obesidad, la anemia y varias deficiencias de vitaminas y minerales. La obesidad conduce a mayor riesgo de padecer diabetes, enfermedades cardiovasculares y otras enfermedades crónicas que provocan muertes prematuras y pérdida de años de vida saludable, y aumentan la demanda de servicios de salud de alto costo. La anemia y la deficiencia de vitaminas y minerales tienen efectos negativos en la salud y el desempeño reproductivo de las mujeres. Cuando estos problemas se presentan durante la gestación, aumentan el riesgo de nacimientos prematuros, con bajo peso al nacer, así como el de anemia y de deficiencia de vitaminas y minerales en el recién nacido. Además, la anemia severa aumenta el riesgo de muertes maternas.

La obesidad es sin duda el problema más preocupante en este grupo de edad. El $52 \%$ de las mujeres fueron clasificadas como con sobrepeso $\mathrm{u}$ obesidad.

La anemia se presentó en $20 \%$ de las mujeres no embarazadas y en $26 \%$ de las embarazadas, la deficiencia de hierro y vitamina $C$ en más de $40 \%$ y la de zinc en $30 \%$. Se encontró también deficiencia en ácido fólico, la cual se asocia con malformaciones congénitas graves, como los defectos del tubo neural.

Se recomienda redoblar esfuerzos de combate a la desnutrición en los programas de la Secretaría de Salud, (Programa de Educación, Salud y Alimentación, Programa de Salud y Nutrición para Pueblos Indígenas, y Arranque Parejo), y en los de distribución de leche y otros alimentos de la Secretaría de De- sarrollo Social, e iniciar acciones vigorosas para atacar el problema de obesidad.

Para información adicional consulte la página del Instituto Nacional de Salud Pública: www.insp.mx/ enn, en donde podrá obtener como documento PDF la publicación completa de la encuesta. Quienes estén interesados en adquirir el libro se pueden comunicar al siguiente correo electrónico: tshamah@insp.mx.

Juan Rivera Dommarco, Teresa Shamah Levy.

Centro de Investigación en Nutrición y Salud, Instituto Nacional de Salud Pública, Cuernavaca, M orelos, M éxico.

\section{REFERENCIAS}

1. Rivera-D ommarco J, Shamah-Levy T, Villalpando-Hernández S, G onzález-de C ossío T, Hernández-Prado B, Sepúlveda J. Encuesta $\mathrm{N}$ acional de Nutrición 1999. Estado nutricio de niños y mujeres en México. Cuernavaca, Morelos, México: Instituto $\mathrm{N}$ acional de Salud Pública, 2001.

\section{Se llevó a cabo la conferencia sobre embarazo no deseado y aborto inducido}

Del 12 al 14 de noviembre de 2001, organizada por el Population Council, la Academia de Medicina de México y el Instituto Alan Guttmacher, se llevó a cabo en las instalaciones del Instituto Nacional de Salud Pública en la ciudad de Cuernavaca, Morelos, México, la conferencia "El embarazo no deseado y el aborto inducido: desafíos de salud pública en América Latina y el Caribe." Los objetivos específicos que la conferencia perseguía eran 1) compartir los resultados de esfuerzos actuales de estudios de investigación; 2) fortalecer la red de investigadores en el tema de embarazo no deseado y aborto indu- 
cido en América Latina y el Caribe, y 3) mejorar la capacidad de difusión de resultados de investigación.

Una de las razones que motivó la realización de dicho evento fue, según la Organización Mundial de la Salud, que la Región de América Latina tiene una alta tasa de embarazos no deseados y la tasa de abortos inseguros más alta del mundo. A pesar de la existencia de estos problemas, poco se sabe sobre las características del embarazo no deseado y del aborto inducido, los factores biológicos, sociales y culturales que influyen sobre ellos, sus conse- cuencias sobre la salud y sus implicaciones para los sistemas de salud. Fue por ello que se organizó esta reunión científica internacional, que permitió compartir y discutir los resultados de más de 100 proyectos de investigación.

Los temas abordados incluyeron: la planificación familiar para reducir el número de abortos, la práctica del aborto legal (con medicamentos y con instrumentos), revisión de las legislaciones, y perspectivas sobre el aborto desde el punto de vista de los hombres y de los profesionales de salud. Asistie- ron casi 300 participantes, que representaron a 18 países de América Latina y el Caribe y de otras partes del mundo. En el último día se realizó un taller sobre cómo informar mejor los resultados de la investigación a diferentes audiencias. Los participantes calificaron el evento de un rotundo éxito, por el contenido desarrollado y por los objetivos cumplidos.

Henry Espinoza N icaragua. Staff Program Associate México, D.F. México. Population Council 